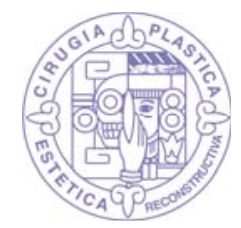

Artículo editorial

\title{
La enseñanza de la Cirugía Plástica
}

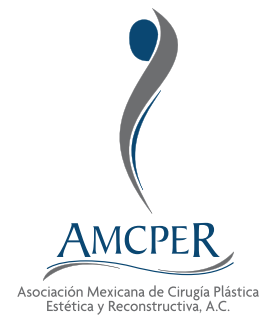

\author{
The teaching of plastic surgery
}

\section{Dr. André Víctor Baldin*}

$\mathrm{M}$ ucho se ha discutido con respecto a la enseñanza de la cirugía plástica a través del tiempo y de las diferentes generaciones, incluso en artículos publicados en números anteriores en esta revista. Se trata de una condición dinámica, que se modifica constantemente, principalmente por tres factores: cambios en la situación de los sistemas de salud, renovación del conocimiento e innovación tecnológica.

Los nuevos desafíos y el crecimiento exponencial de nuestra especialidad y su subdivisión en diferentes áreas del conocimiento tales como mano, craneofacial, microcirugía, cirugía plástica pediátrica, entre otras crean un reto para la enseñanza de la cirugía plástica actual. ${ }^{1}$

La enseñanza de la cirugía plástica debería ser iniciada desde temprano, aún en pregrado. Un modelo sugerido, ya aplicado en algunas universidades internacionales, es el de que los estudiantes de la carrera de médico cirujano tengan una materia asignada a Cirugía Plástica, Estética y Reconstructiva, como mínimo, de uno a dos semestres, durante el ciclo clínico de la carrera (entre el quinto y octavo semestres) como lo hacen con disciplinas como Urología u Ortopedia, por ejemplo. De esa manera, el médico general, interesado o no en realizar la especialidad en cirugía plástica, adquiere conocimientos básicos de la especialidad y lo más importante, conoce sus áreas de actuación, siendo un formador de opinión y de información a colegas de otras áreas y también a pacientes. $^{2}$

La educación del estudiante en postgrado, durante la residencia médica, debe ser lo más amplia posible, como lo marca el Plan Único de Especializaciones Médicas (PUEM), abarcando todas las áreas de su conocimiento y de actuación del Cirujano Plástico, bajo el régimen del Sistema Nacional de Residencias Médicas conforme lo marca la Norma Oficial
Mexicana «NOM-001-SSA3-2012» en materia de Educación en Salud.

La Especialidad en Cirugía Plástica debe ser dinámica, avanzar en la búsqueda constante de nuevos conocimientos, estar en la vanguardia con innovaciones tecnológicas, nuevas técnicas y la búsqueda continua por mejores resultados. ${ }^{3}$

En un ambiente donde los cirujanos plásticos compiten por un mercado lleno de «profesionistas» que realizan procedimientos quirúrgicos, sin tener la capacitación ni el entrenamiento académico y profesional adecuado, es muy poco contentarnos únicamente con la formación técnica del cirujano plástico. Es necesario que la formación científica sea estimulada. ${ }^{4}$ En México se publican al año 17,662 artículos científicos; ocupa el segundo lugar en Latinoamérica después de Brasil y el número 28 a nivel mundial. Actualmente, se realizan 324 publicaciones anuales en revistas médicas. La cantidad de publicaciones en cirugía plástica en México son 27 al año, lo cual nos coloca entre las especialidades con menor número de manuscritos en revistas. Es necesario que los cirujanos plásticos formados en los servicios avalados por el Consejo y la Asociación Mexicana de Cirugía Plástica, Estética y Reconstructiva sean capaces de realizar investigación científica de calidad, la cual debe ser considerada como elemento fundamental de la capacitación del cirujano plástico. ${ }^{5}$

Actualmente, las nuevas generaciones, nos enfrentamos a los nuevos desafíos de nuestra especialidad, incluyendo la posibilidad de una «invasión» de nuestro campo de actuación, como la competencia con otras especialidades, que hoy en día pelean por mismos procedimientos, como ocurre con las especialidad Maxilofacial, de Otorrinolaringología, en la que actualmente se titulan especialistas en Plástica Facial, o los Oftalmólogos con su subespecia- 
lidad en Óculo-Plástica, por ejemplo. Si no hacemos algo como gremio, como asociación, correros el alto riesgo de ver en un futuro próximo la desvalorización de nuestra área de actuación y de nuestra profesión, por lo que las medidas de protección y defensa de la Cirugía Plástica son fundamentales. ${ }^{6}$

En conclusión, la enseñanza de la cirugía plástica debe ser amplia y continua, empezando desde temprano, en la educación de pregrado, proporcionándole a los médicos generales las los conceptos básicos de nuestra especialidad y enseñándoles las áreas de acción del cirujano plástico. Posteriormente, la formación del médico residente debe ser amplia y sólida, basada en aspectos técnicos/quirúrgicos, pero también en su desarrollo científico. Y por último, y no menos importante, la educación médica continua para los cirujanos plásticos egresados formados en los servicios avalados por el Consejo y la Asociación Mexicana de Cirugía Plástica, Estética y Reconstructiva mediante congresos y simposios de calidad. ${ }^{7,8}$

\section{REFERENCIAS}

1. Jacobs DO. Cut well, sew well, do well? N Engl J Med 2013; 369 (15): 1466-1467. http://dx.doi. org/10.1056/NEJMe1309785.
2. Kempton SJ, Bentz ML. Making master surgeons out of trainees: Part I. Teaching surgical judgment. Plast Reconstr Surg 2016; 137 (5): 1646-1653. doi: 10.1097/PRS.0000000000002082. doi: http://dx.doi. org/10.1097/PRS.0000000000002082.

3. Ozturk S, Karagoz H, Zor F. The future of plastic surgery: surgeon's perspective. J Craniofac Surg 2015; 26: e708713. doi: 10.1097/SCS.0000000000002204. doi: http://dx.doi.org/10.1097/ SCS.0000000000002204.

4. Knox AD, Gilardino MS, Kasten SJ, Warren RJ, Anastakis DJ. Competency-based medical education for plastic surgery: where do we begin? Plast Reconstr Surg 2014; 133 (5): 702e-710e. doi: 10.1097/ PRS.0000000000000082. doi: http://dx. doi. org/10.1097/PRS.0000000000000082.

5. Rodríguez WF. Disciplina y autoridad: elementos en la formación de médicos especialistas profesionales. Med Int Mex 2016; 32 (1): 115-117.

6. Montano PJ et al. Impacto da monitoria de Cirurgía Plástica no conhecimento teórico e nas habilidades práticas. Rev Bras Cir Plast 2015; 30 (1): 82-85.

7. Vilchis-López R y cols. Estrategia para incrementar el número de publicaciones científicas en residencias médicas. Cir Plast 2017; 27 (3): 99-106.

8. Trigos MI. La manzana de la discordia. Cir Plast 2015; 25 (1): 3-5.

Correspondencia:

Dr. André Víctor Baldin

Hospital General «Dr. Manuel Gea González», $2^{\circ}$ Piso-Cirugía Plástica y Reconstructiva Calzada de Tlalpan Núm. 4800,

Tlalpan, 14080, Ciudad de México, México.

Teléfono: 4000 3000, ext. 3721

E-mail: andre.baldin03@gmail.com 